\title{
Immunoblockage of 9-0-Acetyl GD3 Ganglioside Arrests the In Vivo Migration of Cerebellar Granule Neurons
}

\author{
Marcelo F. Santiago, Marcos R. Costa, and Rosalia Mendez-0tero \\ Instituto de Biofísica Carlos Chagas Filho, Universidade Federal do Rio de Janeiro, Rio de Janeiro, RJ 21941-590, Brazil
}

\begin{abstract}
During development of the cerebellum, radial glial cells guide the migration of granule cell precursors from the external granular cell layer toward the internal granular cell layer. The cellular membranes of migrating neurons and glial fibers organize a specialized migration junction at the site of contact between these cells, and several molecules have been implicated in the control of this glial-guided neuronal migration program. The monoclonal antibody Jones (mAb Jones) recognizes the ganglioside 9 - $O$-acetyl GD3, which is expressed in migratory profiles in the developing and adult CNS. Recently, this ganglioside was suggested to play a role in neuronal migration in cerebellar cultures. In this report, we use antibody perturbation assays to investigate a possible role of $9-0$-acetyl GD3 in the neuronal migration program in vivo. The results show that chronic intracerebroventricular administration of $\mathrm{mAb}$ Jones arrests neuronal migration in the developing cerebellum of live animals. Proliferating granule cell precursors were labeled with 5-bromo-2'-deoxyuridine (BrdU), and their migratory behavior was analyzed and compared with control groups. Immunoblockage of 9-O-acetyl GD3 arrests 43\% of the BrdU-labeled granule precursors in the external granular cell layer. Together with our previous results, this report strongly suggests that the ganglioside 9-O-acetyl GD3 plays a crucial role in the migration of cerebellar granule cells along radial glial fibers in the developing rat cerebellum.
\end{abstract}

Key words: neuronal migration; radial glia; cerebellum; development; 9-O-acetyl GD3; gangliosides

\section{Introduction}

For more than a century, the cerebellar cortex has been an important model for studies concerning cortical histogenesis (Ramón y Cajal, 1890). Neuronal migration is a basic cellular event essential for the precise integration of neurons in their correct destination place in the vertebrate brain (Sidman and Rakic, 1973; Rakic, 1988, 1990).

During the development of the cerebellum, progenitor cells leave the rhombic lip and migrate tangentially to the neural tube over the surface of the cerebellar anlagen (Miale and Sidman, 1961) (for review, see Wingate, 2001). These progenitor cells undergo several divisions and accumulate under the pia of the developing cerebellum to form the external granular layer (EGL) (Wingate and Hatten, 1999). Once formed, subsequent divisions within the EGL give rise to postmitotic granule cells, which then migrate radially into the cerebellar cortex to form the internal granular layer (IGL) (Hatten, 1990; Rakic, 1990) (for review, see Hatten and Heintz, 1995). The radial migration of granule cells toward the IGL is guided by a specialized form of radial glial cell, the so-called Bergman glia (Sidman and Rakic, 1973; Hatten,

Received March 6, 2003; revised 0ct. 27, 2003; accepted 0ct. 29, 2003.

This work was supported by Programa de Nucleos de Excelencia, Conselho Nacional de Desenvolvimento Científico e Tecnológico, and Fundaçäo Carlos Chagas Filho de Amparo à Pesquisa do Estado do Rio de Janiero grants (R.M.-0.) and by a grant from the Ministry of Science and Technology (M.C.T.) to the Millennium Institute for Tissue Bioengineering (Brazil). We thank Dr. Leny A. Cavalcante and Cecilia Hedin-Pereira for helpful comments and suggestions on this manuscript and Camila Zaverucha-do-Valle and Fernanda Gubert for help with the quantitative analysis.

Correspondence should be addressed to Dr. Marcelo F. Santiago, Instituto de Biofísica Carlos Chagas Filho, Centro de Ciências de Saúde, Bloco G, Cidade Universitária, RJ 21941-590, Rio de Janeiro, Brazil. E-mail: felipe@biof.ufrj.br. DOI:10.1523/JNEUROSCI.0116-03.2004

Copyright $\odot 2004$ Society for Neuroscience $\quad$ 0270-6474/04/240474-05\$15.00/0
1993, 1999). During the glial-guided neuronal migration, a specialized migration junction, an interstitial junction, is present beneath the cell soma at the site of apposition with the glial fiber (Gregory et al., 1988), and this close apposition suggests that membrane components of the cell surface mediate this migration.

Several molecules have been proposed to be involved in glialguided neuronal migration in the developing mammalian CNS (for review, see Pearlman et al., 1998; Hatten, 1999). Recently, a cell surface glycolipid, the ganglioside 9-O-acetyl GD3, has been implicated in gliophilic neuronal migration. In antibody perturbation assays, it was demonstrated that the immunoblockage of this specific ganglioside with the monoclonal antibody Jones (mAb Jones) (Constantine-Paton et al., 1986) arrests neuronal migration in cerebellar slices and reduces the rate of neuronal migration by $\sim 66 \%$ in cerebellar micro explants (Santiago et al., 2001), suggesting that 9-O-acetyl GD3 is necessary for neuronal migration in cerebellar cultures.

In the present work, we tested in vivo whether the ganglioside 9-O-acetyl GD3 has a role in neuronal migration in the rat developing cerebellum. Early postnatal rats received sequential intraventricular injections of $\mathrm{mAb}$ Jones, and the proliferating neuroblasts in the EGL were labeled with intraperitoneal injections of 5-bromo-2'-deoxyuridine (BrdU). This method allowed us to evaluate the directional movement of granule cell precursors in the developing cerebellum and the putative role of the ganglioside 9-O-acetyl GD3 in this phenomenon.

\section{Materials and Methods}

Animals. Lister rats were obtained from our breeding colony. All experiments were performed in accordance with the National Institute of 

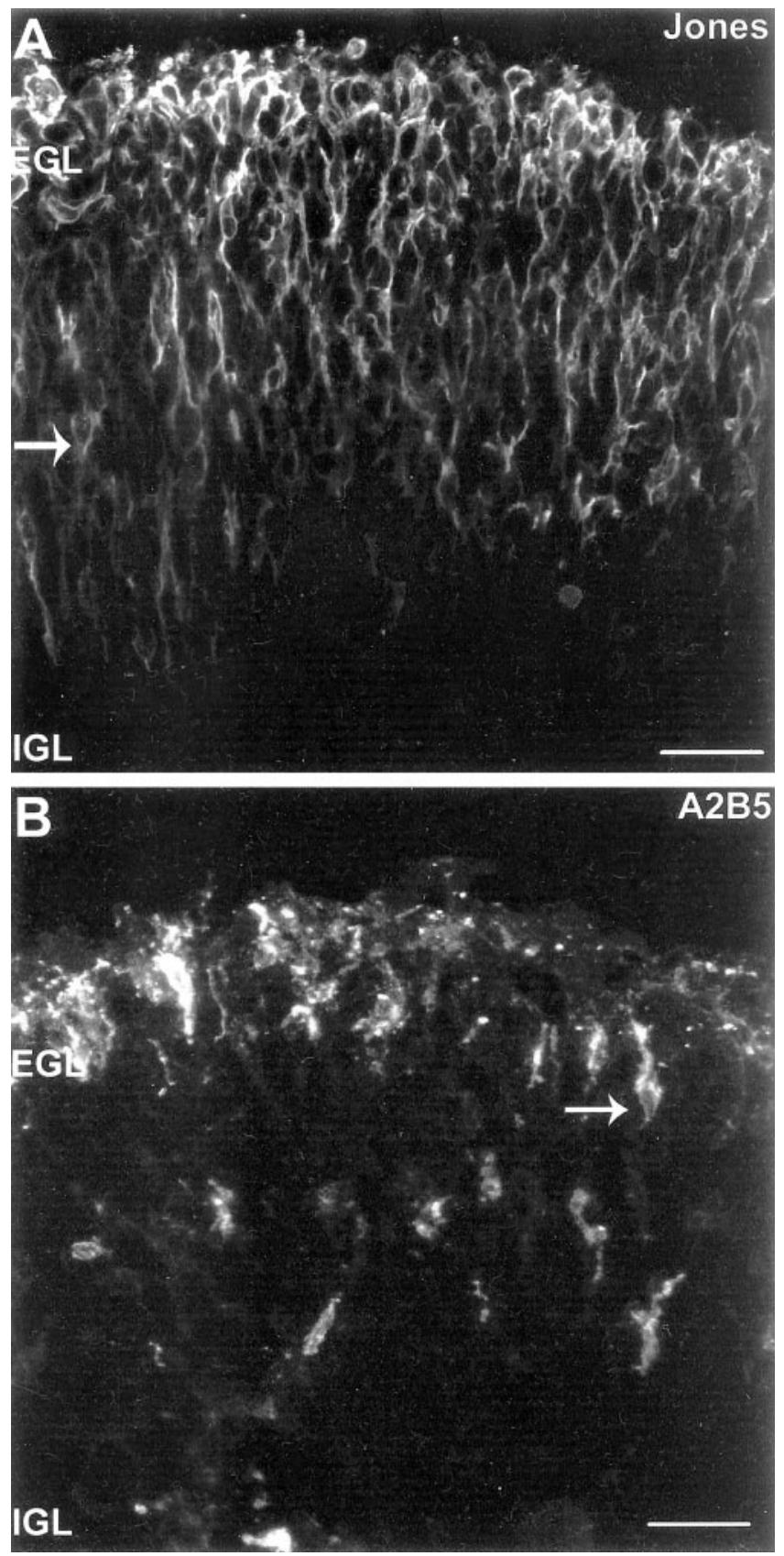

Figure 1. Confocal images of a sagittal section of a P9 postnatal rat cerebella injected with $\mathrm{mAb}$ Jones or A2B5 in the lateral ventricle. The staining was revealed using only a Cy3conjugated secondary antibody specific for mouse lgM. A, Confocal projection of a cerebellar section taken from an mAb Jones-injected animal showing several cells positive for the antibody and displaying migratory profiles leaving the EGL. Arrow points to a bipolar migrating neuron with their leading and trailing process positive for the 9-0-acetyl GD3. B, Confocal projection of a cerebellar section from an $\mathrm{mAb} A 2 \mathrm{~B} 5$-injected animal showing the staining pattern with this antibody. Arrow points to a cell with migratory profile positive for A2B5. Scale bars, $30 \mu \mathrm{m}$.

Health Guide for the Care and Use of Laboratory Animals, and the experimental protocols were approved by the Committee for the Use of Experimental Animals of our Institution.

Intraventricular injections. Postnatal day 7 (P7) rats were briefly anesthetized by ether inhalation and injected, with the assistance of a microsyringe, with $20 \mu \mathrm{l}$ of mAb Jones (IgM; Sigma, St. Louis, MO), mAb A2B5 (IgM; Boehringer Mannheim, Mannheim, Germany), or a sterile $0.9 \%$ saline. The needle was introduced in the posterior portion of the right lateral ventricle. Each animal was injected for 3 consecutive days at a $12 \mathrm{hr}$ interval (a total of five injections) with $\mathrm{mAb}$ Jones $(n=6), \mathrm{mAb}$ A2B5 $(n=4)$, or saline solution $(n=3)$. The mAb Jones specifically recognizes the ganglioside 9-O-acetyl GD3 (Schlosshauer et al., 1988), and $\mathrm{mAb}$ A2B5 recognizes a group of the c-series gangliosides (Eisenbarth et al., 1979) and was used as a control Ig in our experiments.

Bromodeoxyuridine labeling and detection. Newly generated neurons were labeled with BrdU (Sigma), which is incorporated by cells synthesizing DNA (Gratzner, 1982). Thirty minutes before each intraventricular injection, the animals received a dose of $\mathrm{BrdU}(100 \mathrm{mg} / \mathrm{kg}$, i.p., dissolved in $0.007 \% \mathrm{NaOH}$ in saline) and were killed $12 \mathrm{hr}$ after the last intraventricular injection. Control animals from the same offspring were also injected with the drug $(n=5)$. All of the experimental groups were processed for immunohistochemistry as described below. Briefly, the animals were perfused through the ascending aorta with 4\% paraformaldehyde in $0.1 \mathrm{~m}$ phosphate buffer at $\mathrm{pH}$ 7.4. The cerebellum was then removed, cryoprotected by immersion in $20 \%$ phosphate-buffered sucrose, sectioned at $14 \mu \mathrm{m}$, and collected onto gelatin-coated slides. After antigen retrieval in a microwave oven (Dover and Patel, 1994), sections were washed with PBS and incubated overnight at $4^{\circ} \mathrm{C}$ with a mouse monoclonal antibody (IgG) against BrdU (Amersham Biosciences, Sao Paulo, Brazil), according to the instructions of the manufacturer, and developed with a Cy3-conjugated goat anti-mouse $\gamma$-chain specific IgG (1:500; Jackson ImmunoResearch, West Grove, PA). Some sections were also labeled with a polyclonal antibody against GFAP (1:400; Dako, Renteria, CA) and goat anti-rabbit secondary antibody conjugated to Alexa 488 (1:200; Molecular Probes, Eugene, OR). The sections were then labeled with the fluorescent dye 4'-6-diamidino-2-phenylindole (DAPI) (Sigma, S. Louis, MO) or TO-PRO-3 (1:100; Molecular Probes) to visualize the nuclei and mounted in VectaShield (Vector Laboratories, Burlingame, CA). The stained cells were viewed and photographed under a Zeiss (Oberkochen, Germany) Axivert microscope equipped with a Zeiss AxioCam digital color camera connected to the Zeiss AxioVision 3.0 system. The extent of cell migration and the layer-specific localization of BrdU-labeled cells in the cerebellar cortex were analyzed.

In vivo immunohistochemistry. To analyze the diffusion and binding of the injected antibodies (mAb Jones and mAb A2B5), thick cryosections ( $40 \mu \mathrm{m}$; as described above) were incubated for $2 \mathrm{hr}$ at room temperature with a Cy3-conjugated $\mu$-chain-specific goat anti-mouse (1:1000; Jackson ImmunoResearch). The sections were then counterstained with DAPI (Sigma) or TO-PRO-3 (Molecular Probes) and mounted in VectaShield. Three-dimensional projection reconstructed images $(20-40 \mu \mathrm{m})$ were obtained using an LSM 510 Meta Zeiss confocal microscope. In initial experiments, to determine the availability of the antibodies in the brains, the animals received a single intraventricular injection of the antibody (Jones or A2B5) and were allowed to survive for 4, $8,12,16$, or $24 \mathrm{hr}$ after the injection. Animals were fixed, and cryosections were then obtained and analyzed for positive staining as described above. After the 4, 8, or $12 \mathrm{hr}$ survival times, the staining was very strong in the cerebellum, whereas the $16 \mathrm{hr}$ time was weak and absent $24 \mathrm{hr}$ after the injection. Therefore, the interval of $12 \mathrm{hr}$ was chosen for the intraventricular injections.

Quantitative analysis. Identical cerebellar regions (folia) with reference to the dorsoventral, anteroposterior, and mediolateral axes were used to compare the number of BrdU-labeled cells in the different animals. Three-dimensional projection reconstructed images $(14 \mu \mathrm{m})$ were obtained using an LSM 510 Meta Zeiss confocal microscope and analyzed using the Zeiss AxioVision 3.0 system. BrdU-stained cells were counted in the presumptive molecular layer (ML), Purkinje cell layer (PCL), and IGL of injected and control rats. In each experimental group, four adjacent cryostat sections were counted. The data from different experimental conditions were acquired using the morphometric tools of the Zeiss AxioVision 3.0 system. Statistical analysis was performed by ANOVA and the Tukey's multiple comparison post hoc test.

\section{Results}

The pattern of diffusion and staining of intraventricularly injected antibodies against gangliosides was evaluated incubating whole-brain cryosections of the injected animals with Cy3conjugated secondary antibodies. In animals injected with the 

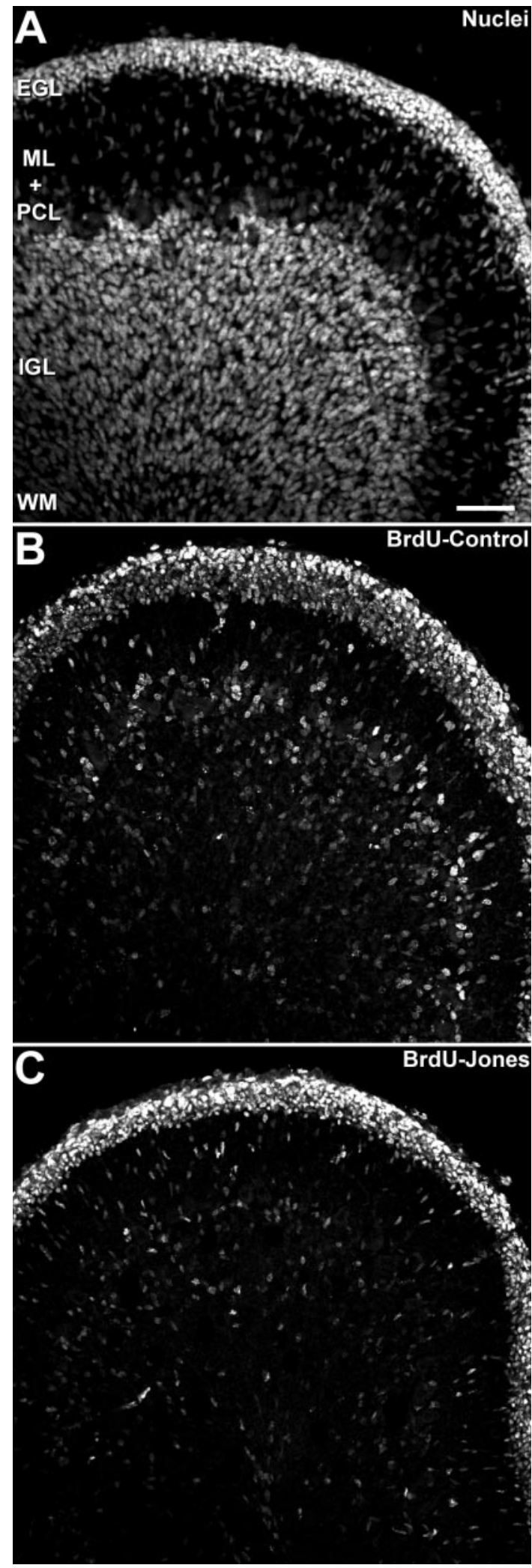

Figure 2. Confocal projections of cerebellar sections showing the effect of $m A b$ Jones on granule cell migration. $A$, Cerebellar section of a control animal stained with the nuclear dye TO-PRO-3 to identify the cerebellar layers. $B$, Cerebellar section from a control animal stained with the anti-BrdU antibody showing the normal pattern of granule cell migration. Observe that several BrdU-positive cells have already reached the inner cerebellar layers. C, Cerebellar section from an animal that received an intraventricular injection of $\mathrm{mAb}$ Jones stained with the antiBrdU antibody. Note that the number of BrdU-positive cells in the inner cerebellar layers is decreased. WM, White matter. Scale bar, $50 \mu \mathrm{m}$.

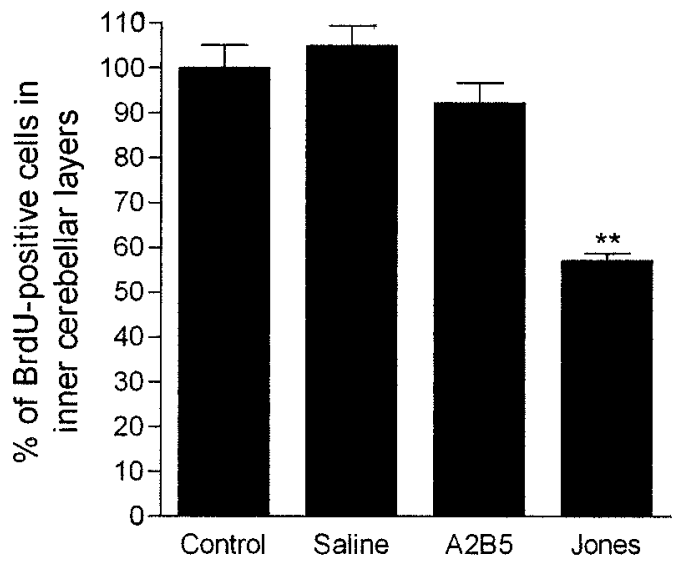

Figure 3. Quantitative analysis of the immunoblockage effect of mAb Jones on neuronal migration in vivo. Data shown represents mean \pm SEM for each group. Intraventricular injection of $\mathrm{mAb}$ Jones decreased by $57 \pm 4 \%(n=6)$ the number of BrdU stained cells in inner cerebellar layers (ML plus PCL plus IGL) compared with the control group (100 $\pm 2 \% ; n=5$ ). Saline injected ( $105 \pm 6 \% ; n=3)$ or mAb A2B5 $(92 \pm 6 \% ; n=4)$ did not have any significant effect on neuronal migration of BrdU-labeled cells when compared with the control group. ${ }^{*} p<0.01$.

antibody against 9-O-acetyl GD3 4-12 hr before fixation, we found immunolabeled cells restricted to very few regions of the brain. These regions include the subventricular zone, the rostral migratory stream in the forebrain, and the cerebellum. The staining pattern of $\mathrm{mAb}$ Jones in the cerebellum of a P9 animal is illustrated in Figure $1 A$. Both the EGL and the presumptive ML display an intense staining in these animals. We also observe in this figure cells leaving the EGL with migratory profiles and expressing the 9-O-acetyl GD3 ganglioside in the cell bodies and also in the trailing and leading processes. In addition, a few cells in the IGL were also stained with mAb Jones. This pattern of staining of 9-O-acetyl GD3 using an in vivo immunohistochemistry was very similar to the expression pattern of 9-O-acetyl GD3 reported previously using immunogold histochemistry (Mendez-Otero et al., 1988) and electron microscope immunohistochemistry (Santiago et al., 2001) techniques.

In animals injected with the A2B5 antibody, the staining pattern was more diffuse than that described in the animals injected with Jones, and A2B5-positive cells were observed in all cerebellar layers (Fig. $1 B$ ). Some of the A2B5-labeled cells also display a migratory profile. No staining was found in the cerebellum or in any other brain region in saline-injected and control animals.

The intraventricularly injected and control animals received several intraperitonial injections of BrdU to label the cells in proliferation and to allow us to follow the postmitotic cell migration and to analyze the layer-specific localization of the BrdU-labeled cells in the cerebellar cortex. In control animals, several BrdUlabeled cells were found in the EGL, in the presumptive ML, PCL, and IGL (inner cerebellar layers) (Fig. 2B). A similar pattern of BrdU staining was observed in the cerebella of animals injected intraventricularly with saline (data not shown). In animals injected with mAb A2B5, we observe that the presence of the antibody for $3 \mathrm{~d}$ into the brain environment did not cause any significant effect on the extent of postmitotic granule cell migration. In these animals, BrdU-stained cells migrated through all cerebellar layers in a pattern very similar to that described in the cerebellum of control animals and saline-injected ones (data not shown). In contrast, injections of $\mathrm{mAb}$ Jones caused a drastic effect on the migration pattern of granule cells in equivalent folium of the 

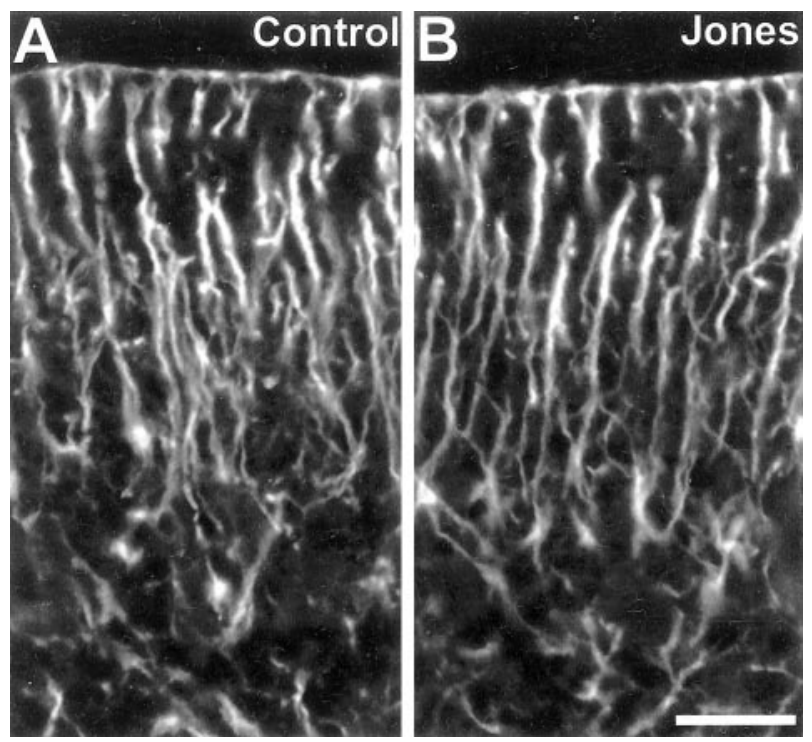

Figure 4. The presence of $\mathrm{mAb}$ Jones does not affect radial glia morphology in the developing cerebellum. $A$, Confocal projection of a cerebellar section of a control animal stained with antibody against GFAP showing the normal pattern of radial glia morphology. $B$, Confocal projection of a cerebellar section of an animal injected with $\mathrm{mAb}$ Jones and stained with antiGFAP showing that the presence of the antibody does not change the normal morphology of the radial glial cells. Scale bar, $30 \mu \mathrm{m}$.

developing cerebellum (Fig. 2C). In these animals, the majority of postmitotic BrdU-labeled cells were arrested in the EGL, and only a small number of BrdU-positive cells reached the inner cerebellar layers (Fig. 2C). The quantitative analysis of these data are represented in Figure 3. The presence of $\mathrm{mAb}$ Jones in the cerebellum decreases by $43 \%$ the number of postmitotic BrdUpositive cells in the inner cerebellar layers when compared with the control groups (Fig. 3).

We tested whether the injected antibody is interfering with the radial glia that gives support to the granule cell migration. The pattern of staining with GFAP in control (Fig. $4 A$ ) and in $\mathrm{mAb}$ Jones-injected animals (Fig. 4) shows similar radial glia profiles, which suggest that the antibody does not affect the morphology of the these cells.

\section{Discussion}

Our results show that the intraventricular injection of $\mathrm{mAb}$ Jones in early postnatal animals labels cells in migratory regions of the developing brain, including the cerebellum. In these regions, cells are found with migratory profiles. The observation that labeling with Jones $\mathrm{mAb}$ is restricted to regions of cell migration is consistent with previous reports that have suggested that the ganglioside 9-O-acetyl GD3 is a marker for neuronal migration in the developing (Mendez-Otero et al., 1988; Schlosshauer et al., 1988; Mendez-Otero and Ramon-Cueto, 1994; Miyakoshi et al., 2001; Santiago et al., 2001) (for review, see Mendez-Otero and Santiago, 2001) and adult (Mendez-Otero and Cavalcante, 1996) nervous system. We also show that the antibody against 9-Oacetyl GD3 arrests granule cells in the external granular cell layer and diminishes the number of BrdU-positive granule cells in the internal granular cell layer by $57 \%$. This effect is not attributable to an interference with the glial radial fibers that support cell migration in this system with the exit of the cells from the cell cycle, because shorter treatments with the antibody do not affect cell migration and proliferation (Santiago et al., 2001).

Recently, it has been proposed that gangliosides and other glycolipids are present in specialized membrane microdomains named "glycosynapses" (Hakomori, 2002). It has also been suggested that these molecular assemblies can mediate cell adhesion and/or signal transduction events through tetraspanin-integringanglioside complexes (Zheng et al., 1993; Mannion et al., 1996; Hemler, 1998; Ono et al., 2000, 2001; Wang et al., 2001). In addition, it has been shown that $\alpha 3 \beta 1$ integrin plays a key role in the control of glial-guided neuronal migration during the formation of the cerebral cortex layers (Dulabon et al., 2000), and $\beta 1$ integrin expression has been described in the cerebellum during development (Husmann and Sievers, 1985). These results combined with results described in this paper and previous reports from our group (for review, see Mendez-Otero and Santiago, 2001) support the assumption that 9-O-acetyl GD3 may interact with integrin receptors and therefore be involved in the control and/or modulation of the neuron-glia interactions and, consenquently, in the neuronal migration program during cerebellar development. Because gangliosides have been associated with glycosynapses in non-neuronal cells, it would be interesting to verify whether such structures are also present in the developing cerebellum and their role in the gliophilic migration of granule cells.

\section{References}

Constantine-Paton M, Blum AS, Mendez-Otero R, Barnstable CJ (1986) A cell surface molecule distributed in a dorsoventral gradiente in the perinatal rat retina. Nature 324:459-462.

Dover R, Patel K (1994) Improved methodology for detecting bromodeoxyuridine in cultured cells and tissue sections by immunocytochemistry. Histochemistry 102:383-387.

Dulabon L, Olson EC, Taglienti MG, Eisenhuth S, McGrath B, Walsh CA, Kreidberg JA, Anton ES (2000) Reelin binds alpha3betal integrin and inhibits neuronal migration. Neuron 27:33-44.

Eisenbarth GS, Walsh FS, Nirenberg M (1979) Monoclonal antibody to a plasma membrane antigen of neurons. Proc Natl Acad Sci USA 76:4913-4917

Gratzner HG (1982) Monoclonal antibody to 5-bromo and 5-iododeoxyuridine: a new reagent for detection of DNA replication. Science 218:474-478.

Gregory WA, Edmondson JC, Hatten ME, Mason CA (1988) Cytology and neuron-glial apposition of migrating cerebellar granule cells in vitro. J Neurosci 8:1728-1738.

Hakomori SI (2002) The glycosynapse. Proc Natl Acad Sci USA 99:225-232. Hatten ME (1990) Riding the glial monorail: a common mechanism for glial-guided neuronal migration in different regions of the developing brain. Trends Neurosci 13:179-187.

Hatten ME (1993) The role of migration in central nervous system neuronal development. Curr Opin Neurobiol 2:38-44.

Hatten ME (1999) Central nervous system neuronal migration. Annu Rev Neurosci 22:511-539.

Hatten ME, Heintz N (1995) Mechanisms of neural patterning and specification in the developing cerebellum. Annu Rev Neurosci 18:385-408.

Hemler ME (1998) Integrin associated proteins. Curr Opin Cell Biol 10:578-585.

Husmann B, Sievers J (1985) Cerebellar external granule cells are attached to the basal lamina from the onset of migration up to the end of their proliferative activity. J Comp Neurol 241:50-62.

Mannion BA, Berditchevski F, Kraeft SK, Chen LB, Hemler ME (1996) Transmembrane-4 superfamily proteins CD81 (TAPA-1), CD82, CD63, and CD53 specifically associated with integrin alpha 4 beta 1 (CD49d/ CD29). J Immunol 157:2039-2047.

Mendez-Otero R, Cavalcante L (1996) Expression of 9-O-acetylated gangliosides is correlated with tangential cell migration in the rat brain. Neurosci Lett 204:97-100.

Mendez-Otero R, Ramon-Cueto A (1994) Expression of 9-O-acetylated gangliosides during development of the rat olfactory system. NeuroReport 5:1755-1759.

Mendez-Otero R, Santiago MF (2001) Functional role of a glycolipid in directional movements of neurons. An Acad Bras Cienc 73:221-229. 
Mendez-Otero R, Schlosshauer B, Barnstable CJ, Constantine-Paton M (1988) A developmentally regulated antigen associated with neural cell and process migration. J Neurosci 8:564-579.

Miale IL, Sidman RL (1961) An autoradiographic analysis of histogenesis in the mouse cerebellum. Exp Neurol 4:277-296.

Miyakoshi LM, Mendez-Otero R, Hedin-Pereira C (2001) The 9-O-acetyl GD3 ganglioside is expressed by migrating chains of subventricular zone neurons in vitro. Braz J Med Biol Res 34:669-673.

Ono M, Handa K, Withers DA, Hakomori S (2000) Glycosylation effect on membrane domain (GEM) involved in cell adhesion and motility: a preliminary note on functional alpha3, alpha5-CD82 glycosylation complex in ldlD 14 cells. Biochem Biophys Res Commun 279:744-750.

Ono M, Handa K, Sonnino S, Withers DA, Nagai H, Hakomori S (2001) GM3 ganglioside inhibits CD9-facilitated haptotactic cell motility: coexpression of GM3 and CD9 is essential in the downregulation of tumor cell motility and malignancy. Biochemistry 40:6414-6421.

Pearlman AL, Faust PL, Hatten ME, Brunstrom JE (1998) New directions for neuronal migration. Curr Opin Neurobiol 8:45-54.

Rakic P (1988) Specification of cerebral cortical areas. Science 241:170-176

Rakic P (1990) Principles of cell migration. Experientia 46:882-891.

Ramón y Cajal S (1890) A propos de certains éléments bipolaries du cervelet avec quelques détails nouveaux sur l'évolution des fibres cérébelleuses. Int Mschr Anat Physiol 7:12-31.

Santiago MF, Berredo-Pinho M, Costa MR, Gandra M, Cavalcante LA, Mendez-Otero R (2001) Expression and function of ganglioside 9-Oacetyl GD3 in granule cell development. Mol Cel Neurosci 17:488-499.

Schlosshauer B, Blum AS, Mendez-Otero R, Barnstable CJ, ConstantinePaton M (1988) Developmental regulation of ganglioside antigens recognized by the JONES antibody. J Neurosci 8:580-592.

Sidman RL, Rakic P (1973) Neuronal migration with special reference to developing human brain: a review. Brain Res 62:1-35.

Wang X, Sun P, Al-Qamari A, Tai T, Kawashima I, Paller AS (2001) Carbohydrate-carbohydrate binding of ganglioside to integrin alpha(5) modulates alpha(5)beta(1) function. J Biol Chem 276:8436-8444.

Wingate RJ (2001) The rhombic lip and early cerebellar development. Curr Opin Neurobiol 11:82-88.

Wingate RJ, Hatten ME (1999) The role of rhombic lip in avian cerebellum development. Development 126:4395-4404.

Zheng M, Fang H, Tsuruoka T, Tsuji T, Sasaki T, Hakomori S (1993) Regulatory role of GM3 ganglioside in alpha 5 beta 1 integrin receptor for fibronectin-mediated adhesion of FUA169 cells. J Biol Chem 268: 2217-2222. 